\title{
On the Bright Rims of $\mathrm{H} \alpha$ Filaments
}

\author{
F. Paletou \\ Observatoire de la Côte d'Azur, Département Cassini, BP 4229, F-06304 \\ Nice Cedex 4, France E-mail: paletou@obs-nice.fr
}

\begin{abstract}
Heinzel et al. (1995) have proposed that diffusive penetration of the incident chromospheric $\mathrm{H} \alpha$ radiation could explain the bright rims of filaments. We examine this suggestion with the help of new 2D radiative models of filaments and show that our computations do not support this explanation.
\end{abstract}

\section{Introduction}

Bright rims of filaments have been observed for decades. Early observations made in $\mathrm{H} \alpha$ at Kodaikanal Observatory were reported by Royds (1920). A systematic investigation based on the study of 271 filaments can be found in the 1948 mémoire of D'Azambuja and d'Azambuja. However, this feature of filaments is not yet well understood. Several scenarios have been used in order to explain bright rims: local heating due to magnetic reconnection at the footpoints (Engvold 1988, Kononovitch et al. 1994), increased H $\alpha$ chromospheric emission in the filament channel because of a lack of "dark structures" (Heinzel et al. 1990), radiative interaction between the prominence and the underlying chromosphere (the so-called "prominence blanketing" effect; see Kostik and Orlova 1975 , Zirin 1988) or diffusive penetration of the incident $\mathrm{H} \alpha$ chromospheric illumination into the filament body (Heinzel et al. 1995).

Using a 1D horizontal slab model, Heinzel et al. (1995) showed that a "bump" in the $\mathrm{H} \alpha$ source function, $\mathrm{S}(\mathrm{H} \alpha)$, can be produced at the bottom of the slab and they interpreted it as the location of the bright rim. Basically, their explanation is that the rather large amount of chromospheric radiation penetrating the slab from below and scattered out could be seen as a bright contrast feature over a limb-darkened $\mathrm{H} \alpha$ chromosphere.

We discuss this latter tentative explanation from the standpoint of twodimensional (2D) radiative modelling of prominences and filaments.

\section{2D Models: Results and Discussion}

Our 2D models use a 5-level hydrogen atom plus a continuum. Ly $\alpha$ and Ly $\beta$ resonance lines are formed with partial frequency redistribution, and realistic incident radiation has been used (Paletou 1995, 1996). We have computed five different homogeneous $2 \mathrm{D}$ slab models with the same input parameters as in Heinzel et al. (1995), i.e., temperature $(8000 \mathrm{~K})$, gas pressure $\left(0.3\right.$ dyn $\left.\mathrm{cm}^{-2}\right)$, vertical (geometrical) extension $(3200 \mathrm{~km})$ and height above the solar surface 

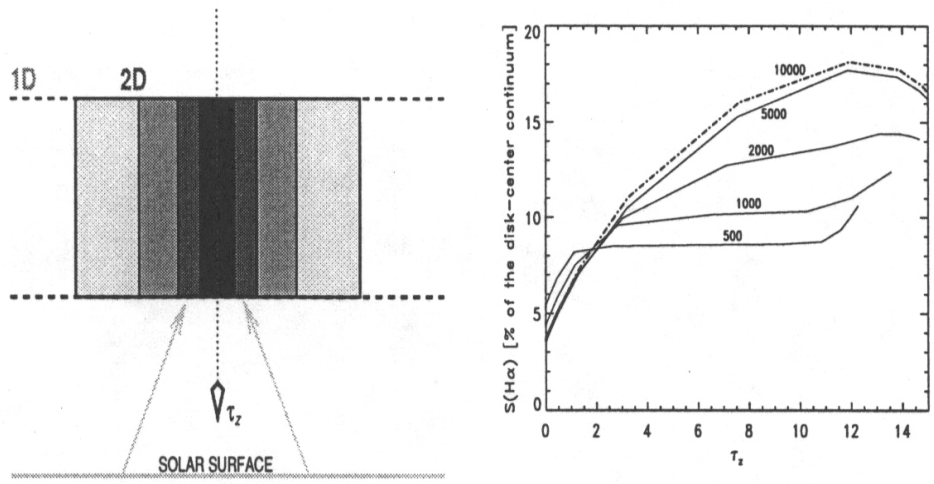

Figure 1. Left: Sketch of the different 2D model geometries we used. Only the geometrical width of the structure was changed from one model to another. Right: Source function variations along the vertical symmetry axis displayed for different geometrical widths in $\mathrm{km}$.

$(10,000 \mathrm{~km})$.

However, unlike in the $1 \mathrm{D}$ case we used different geometrical widths for the filament, namely: $10,000,5000,2000,1000$ and $500 \mathrm{~km}$ (Figure 1). The slab with the largest width is optically thick enough in the horizontal direction to reproduce along the vertical symmetry axis the $1 \mathrm{D} \mathrm{S}(\mathrm{H} \alpha)$ variations (cf., Heinzel et al. 1995, Figure 5). Hereafter, the source functions and emergent intensities will be expressed as a percentage of the disk-center continuum $\left(4.077 \times 10^{-5} \mathrm{cgs}\right)$.

In Figure 1 (right) we show how the vertical variations of $\mathrm{S}(\mathrm{H} \alpha)$ differ when the width of the 2D slab is reduced; each curve is labelled with the corresponding width in $\mathrm{km}$. $\mathrm{S}(\mathrm{H} \alpha)$ ranges between the $1 \mathrm{D}$ solution and the optically thin limit $(\mathrm{S}(\mathrm{H} \alpha) \approx 8 \%$ at all heights).

We also calculated what is actually emerging from the slab for a line of sight (LOS) close to the horizontal direction. Obviously, not only vertical variations but also horizontal ones will drive the emergent line intensity. Needless to say it is impossible to evaluate this using a 1D horizontal slab model, simply because the latter is infinite along the LOS of interest! In Figure 2 (left) we plot the full $2 \mathrm{D}$ variations of $\mathrm{S}(\mathrm{H} \alpha)$ together with (right) the line center emergent intensity $\mathrm{I}_{c}(\mathrm{H} \alpha)$ for a LOS parallel to the horizontal direction. It is important to note that in Figure 2 (right) the height is expressed in geometrical units.

We do not find any pattern in the $\mathrm{I}_{c}(\mathrm{H} \alpha)$ vertical variations which reproduce a bright rim, i.e., a local peak of $\mathrm{H} \alpha$ emission at low altitude. Pushing the investigation further, we computed another set of 2D slab models for higher temperatures, gas pressures, heights and lower altitudes above the surface. Our results are displayed in Figure 3, and from these we conclude that, instead of showing a bright rim, it shows unrealistically bright prominences. 

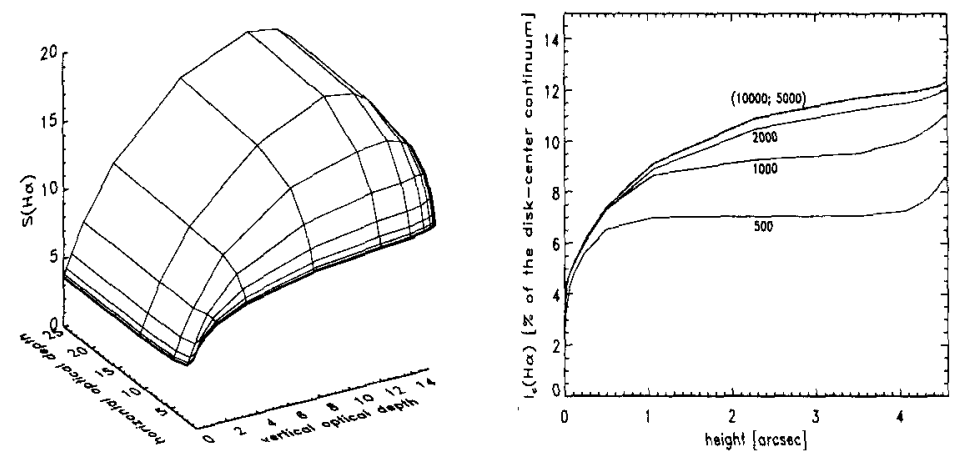

Figure 2. Left: Full 2D variations of the source function; only the half-slab is displayed for symmetry reasons. Right: Vertical variations of the emergent line center intensity along the symmetry axis: results for different geometrical widths in $\mathrm{km}$.
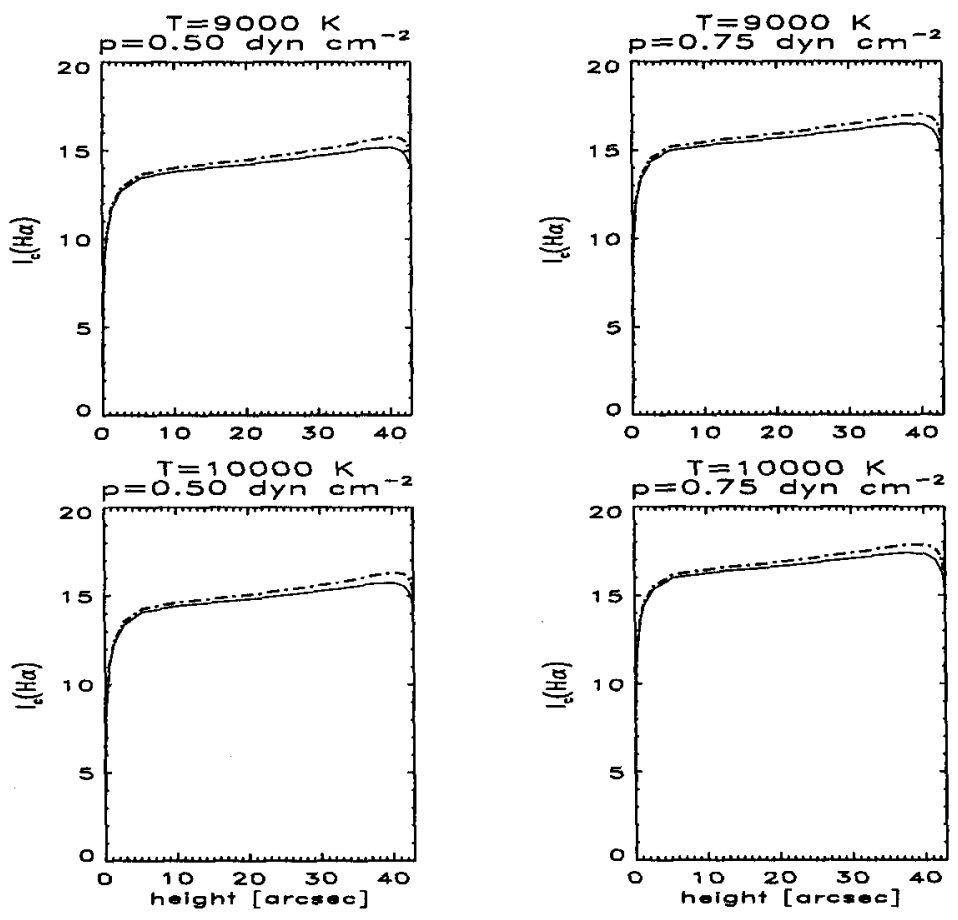

Figure 3. $\mathrm{I}_{c}(\mathrm{H} \alpha)$ vertical variations obtained with higher slab heights, gas pressures and temperatures. We also considered lower altitudes of the $2 \mathrm{D}$ slab above the solar surface (in $\mathrm{km}, 1000$ : dashed lines and 5000: solid lines). 


\section{Conclusion}

We showed how the explanation of an $\mathrm{H} \alpha$ bright rim due to diffusive penetration of the incident radiation - a pure radiative transfer effect - seems to be insufficient when considering isothermal and isobaric 2D models of filaments.

Other explanations such as prominence blanketing should be investigated further by considering in some detail the radiative interaction between a 2D filament and the chromosphere that lies beneath it. It is also very likely that the existence of fine structure elements favour the penetration of the incident radiation to deep layers of the prominence. These two issues can be addressed further in multidimensional geometry.

Here we have demonstrated that significant geometrical effects can occur for a spectral line of moderate optical thickness such as $\mathrm{H} \alpha$. More generally, we consider that diagnostics relying on radiative models need to be improved upon by using results from multidimensional models.

Acknowledgments. FP wishes to thank Drs. J.E. Wiik, R. MolownyHoras and J.A. Tully for their helpful comments on the manuscript.

\section{References}

D'Azambuja L. and d'Azambuja M. 1948, Ann. Obs. Paris-Meudon 6, 7

Engvold O. 1988, in Dynamics and Structures of Quiescent Solar Prominences, E.R. Priest (ed.), Kluwer Acad. Pub., Dordrecht, Holland, p. 47

Heinzel P., Kononovich E. V. and Smirnova O. B. 1990, Debrecen Obs. 7, 172 Heinzel P. et al., 1995, Solar Phys., 160, 19

Kononovich E.V. et al. 1994, in Solar Coronal Structures, V. Rusin et al. (eds.), Astr. Inst., Slovak Republic, p. 365

Kostik R.I. and Orlova T.V. 1975, Solar Phys. 45, 119

Paletou F. 1995, A\&A 302, 587

Paletou F. 1996, A\&A 311, 708

Royds T. 1920, Kodaikanal Obs. Bull. 63, 289

Zirin H. 1988, Astrophysics of the Sun, Cambridge Univ. Press, Cambridge 